\title{
CLOSED IDEALS IN $C(X)$
}

\author{
PHILIP NANZETTA AND DONALD PLANK
}

\begin{abstract}
The characterization of uniformly closed ideals in $C(X)$, for $X$ compact Hausdorff, is well known. In this note, we extend this characterization to an arbitrary completely regular Hausdorff $X$ and derive some corollaries.
\end{abstract}

1. Preliminaries. We shall assume that the reader is familiar with the terminology and basic results of the Gillman and Jerison text [GJ]. Thus, $C(X)$ will denote the algebra of all continuous real-valued functions defined on the space $X$. The class of algebras $C(X)$ is unaltered if we restrict attention to completely regular Hausdorff spaces $X$, and therefore $X$ will always denote a completely regular Hausdorff space in the sequel.

Each continuous $f: X \rightarrow \boldsymbol{R}$ admits a unique continuous extension $f^{*}: \beta X \rightarrow$ $\gamma \boldsymbol{R}$ where $\beta X$ denotes the Stone-Cech compactification of $X$ and $\gamma \boldsymbol{R}$ denotes the extended reals (the two-point compactification of the reals $\boldsymbol{R}$ ). For $I \subseteq C(X)$, we write $I^{*}=\left\{f^{*}: f \in I\right\}$. We shall expand the zero-set notation $Z(f)$ and $Z[I]$ to include extended real-valued functions.

We may define a metric $\rho$ on $C(X)$ by the formula

$$
\rho(f, g)=\sup \{|f(x)-g(x)| \wedge 1: x \in X\} .
$$

This metric is complete, and $C(X)$ becomes a topological vector space, but in general not a topological ring, in the metric topology. This topology is called the uniform topology (or u-topology), and the reader is referred to [H] for further details. If $I \subseteq C(X)$, then $I$ will denote the uniform closure of $I$. In the remainder of this note, all topological properties of $C(X)$ will refer to the uniform topology.

By "ideal", we shall mean "proper ring ideal".

2. Closures of ideals. If $I$ is an ideal in $C(X)$, then its closure $I$ is easily seen to be a proper closed vector sublattice of $C(X)$. However, $I$ need not be an ideal; there may exist $f \in I$ and $g \in C(X)$ such that $f g \notin I$.

Presented to the Society, January 18, 1972; received by the editors December 1, 1971.

AMS 1970 subject classifications. Primary 54C40, 54C50; Secondary 46E25, 46H10.

Key words and phrases. Rings of continuous functions, closed ideal, uniform topology, stationary set, extended real-valued function. 
In fact, the next result guarantees the existence of such an $I$ for any nonpseudocompact space $X$.

2.1 THEOREM. The following conditions are mutually equivalent.

(a) $X$ is pseudocompact.

(b) The closure of any ideal in $C(X)$ is an ideal.

(c) Each ideal in $C(X)$ is contained in a closed ideal.

Proof. (a) implies (b). If $X$ is pseudocompact, then $C(X)=C(\beta X)$ is a topological algebra under the uniform (norm) topology.

(b) implies (c). Clear.

(c) implies (a). Suppose that $X$ is not pseudocompact; thus, there exists an unbounded $f \in C(X)$ which we may assume to be strictly positive. Define

$$
\begin{aligned}
F_{n} & =\{x \in X: f(x) \geqq n\}, \quad n=1,2,3, \cdots, \\
I & =\left\{g \in C(X): F_{n} \subseteq Z(g) \text { for some } n\right\} .
\end{aligned}
$$

Then $I$ is an ideal in $C(X)$. Suppose that $I$ were contained in the closed ideal $J$; we shall show that $1 / f \in J$ in contradiction to the definition of $J$. Let $\varepsilon>0$ ( $\varepsilon$ real) be given, and choose a positive integer $n$ such that $1 / n<\varepsilon$. Define $g=(1 / f-1 / n) \vee 0$. Then $F_{n} \subseteq Z(g)$, so that $g \in I \subseteq J$, while $0<(1 / f)-g$ $=1 / n \wedge 1 / f \leqq 1 / n<\varepsilon$. Hence $1 / f \in J$.

2.2 EXAMPLE. A particular ideal can be contained in a closed ideal without its closure being an ideal. Let $X$ be nonpseudocompact, and let $I$ be the ideal constructed in 2.1. Let $Y$ be the disjoint union of $X$ and the one-point space $\{p\}$, and define

$$
\begin{aligned}
J & =\{f \in C(Y): f \mid X \in I, f(p)=0\}, \\
K & =\{f \in C(Y): f \mid X \in \bar{I}, f(p)=0\}, \\
M & =\{f \in C(Y): f(p)=0\} .
\end{aligned}
$$

Then $J$ is an ideal in $C(Y)$ which is contained in the closed ideal $M$, while $\bar{J}=K$ is not an ideal.

Even though the closure of an ideal $I$ need not be an ideal, we can still give an explicit formula for $I$ (cf. [GJ, 40]).

2.3 THEOREM. If $I$ is an ideal in $C(X)$, then

$$
\bar{I}=\left\{f \in C(X): Z\left(f^{*}\right) \supseteq \bigcap Z\left[I^{*}\right]\right\} .
$$

Proof. Let $I$ be an ideal in $C(X)$, and define $\Delta=\bigcap Z\left[I^{*}\right]$ and $J=$ $\left\{f \in C(X): Z\left(f^{*}\right) \supseteq \Delta\right\}$. Clearly $J$ is closed and $I \subseteq J$. It suffices to show that $J \subseteq I$. So let $f \in J$ and $\varepsilon>0$ ( $\varepsilon$ real) be given. Letting

$$
g=[(f-\varepsilon) \vee 0]+[(f+\varepsilon) \wedge 0],
$$


we have $|f-g| \leqq \varepsilon$ and $Z\left(g^{*}\right) \supseteq f^{* \leftarrow}((-\varepsilon, \varepsilon))$, a neighborhood of $\Delta$. We must show that $g \in I$. By compactness of $\beta X$, there exist $h_{1}, h_{2}, \cdots, h_{n} \in I$ such that $Z\left(g^{*}\right)$ is a neighborhood of $\bigcap_{i=1}^{n} Z\left(h_{i}^{*}\right)$. Defining $h=h_{1}^{2}+$ $h_{2}^{2}+\cdots+h_{n}{ }^{2}$, we have $h \in I$ and $Z\left(h^{*}\right)=\bigcap_{i=1}^{n} Z\left(h_{i}^{*}\right)$. If we let

$$
\begin{aligned}
k(x) & =g(x) / h(x) & & \text { for } x \in X \sim Z(g), \\
& =0 & & \text { for } x \in Z(g),
\end{aligned}
$$

then $k \in C(X)$ and $g=k h \in I$.

2.4 Corollary. An ideal I in $C(X)$ is closed if and only if

$$
I=\left\{f \in C(X): Z\left(f^{*}\right) \supseteq \bigcap Z\left[I^{*}\right]\right\} .
$$

3. Ideal sets. We have shown that a closed ideal in $C(X)$ consists of all functions $f$ whose extensions $f^{*}$ vanish on some fixed nonvoid compact set. Let us now consider the problem in reverse. That is, let $\Delta$ be some nonvoid compact subset of $\beta X$, and form the set $I=\left\{f \in C(X): Z\left(f^{*}\right) \supseteq \Delta\right\}$. Then $I$ is a closed vector sublattice of $C(X)$ but need not be an ideal. For example, let $X=N$, the discrete space of positive integers, and $\Delta=\{p\}$ where $p \in \beta N \sim N$. Then $I$ contains the unit $f$, where $f(n)=1 / n$, even though $I \neq C(X)$. We shall call $\Delta$ an ideal set if $I$ is an ideal.

We now give a topological characterization of ideal sets, but first we need a definition. We shall say that a subset $S$ of $\beta X$ is far from $X$ if there exists a zero-set $Z$ of $\beta X$ such that $S \subseteq Z \subseteq \beta X \sim X$; otherwise $S$ is close to $X$. Thus, $X$ is realcompact if and only if each singleton subset of $\beta X \sim X$ is far from $X[\mathbf{G J}, 8.8]$, and $X$ is Lindelöf if and only if each compact subset of $\beta X \sim X$ is far from $X$ [S]. Note that, by [GJ, 7D(1)],

$$
\operatorname{cl}_{\beta X} Z(f)=\left\{p \in \beta X:(f g)^{*}(p)=0 \text { for all } g \in C(X)\right\}
$$

for $f \in C(X)$.

3.1 THEOREM. The following conditions are mutually equivalent for any nonvoid compact subset $\Delta$ of $\beta X$.

(a) $\Delta$ is an ideal set.

(b) $\Delta=\bigcap Z\left[I^{*}\right]$ for some closed ideal $I$ in $C(X)$.

(c) $Z\left(f^{*}\right) \supseteq \Delta$ implies $\mathrm{cl}_{\beta X} Z(f) \supseteq \Delta$ for all $f \in C(X)$.

(d) If $S$ is far from $X$, then $\mathrm{cl}_{\beta X}(\Delta \sim S)=\Delta$.

Proof. (a) implies (b). Let $I=\left\{f \in C(X): Z\left(f^{*}\right) \supseteq \Delta\right\}$.

(b) implies (c). Suppose that $\Delta=\bigcap Z\left[I^{*}\right]$ for some closed ideal $I$; then by $2.4, I=\left\{f \in C(X): Z\left(f^{*}\right) \supseteq \Delta\right\}$. Let $f \in C(X)$ with $Z\left(f^{*}\right) \supseteq \Delta$. Then $f \in I$, whence $f g \in I$ for all $g \in C(X)$. But then

$$
\operatorname{cl}_{\beta X} Z(f)=\bigcap\left\{Z\left((f g)^{*}\right): g \in C(X)\right\} \supseteqq \Delta .
$$


(c) implies (d). Suppose that $S$ is far from $X$, but there exists $p \in \Delta$ with $p \notin \operatorname{cl}_{\beta X}(\Delta \sim S)$. Then there exist $h, k \in C^{*}(X)$ such that $S \subseteq Z\left(h^{*}\right) \subseteq$ $\beta X \sim X, p \notin Z\left(k^{*}\right)$ and $\Delta \sim S \subseteq Z\left(k^{*}\right)$. Let $f=h k$ and $g=1 / h$. Then $Z\left(f^{*}\right)=Z\left(h^{*}\right) \cup Z\left(k^{*}\right) \supseteq \Delta$, but $p \notin Z\left(k^{*}\right)=Z\left((f g)^{*}\right)$ so that $p \notin \mathrm{cl}_{\beta X} Z(f)$.

(d) implies (a). Suppose that $\Delta$ is not an ideal set. Thus, if we let $I=\left\{f \in C(X): Z\left(f^{*}\right) \supseteq \Delta\right\}$, then there exist $f, g \in C(X)$ such that $f \in I$ and $f g \notin I$. So $Z\left(f^{*}\right) \supseteq \Delta$ and for some $p \in \Delta, p \notin Z\left((f g)^{*}\right)$. Let $Z$ be a zero-set neighborhood of $p$ in $\beta X$ such that $Z \cap Z\left((f g)^{*}\right)=\varnothing$; then $S=\Delta \cap Z$ is far from $X$, since $S \subseteq Z\left(f^{*}\right) \cap Z \subseteq \beta X \sim X$. But $Z$ is a neighborhood of $p$ which does not meet $\Delta \sim S$, so $\operatorname{cl}_{\beta X}(\Delta \sim S) \neq \Delta$.

It follows from 3.1 and 2.4 that every closed ideal is a $z$-ideal and therefore is absolutely convex (i.e. is an $l$-ideal; cf. [P, 3.7]).

It is clear from 3.1 that an ideal set must be close to $X$. The converse, however, does not hold. For example, let $X=N$ and $\Delta=\{1, p\}$ where $p \in \beta N \sim N$. Then $\Delta$ is close to $X$, but is not an ideal set. We do have the following partial converse.

3.2 Lemma. Any compact subset of $\beta X$ which is close to $X$ contains an ideal set.

Proof. Let $K$ be a compact subset of $\beta X$ which is close to $X$, and define $\Delta=\bigcap\left\{\mathrm{cl}_{\beta X} Z(h): Z\left(h^{*}\right) \supseteq K\right\}$, a nonvoid compact subset of $K$. We shall use 3.1(c) to show that $\Delta$ is an ideal set. Thus, suppose that $Z\left(f^{*}\right) \supseteq \Delta$ for some $f \in C(X)$. By the definition of $\Delta$, for each $p \in \beta X \sim Z\left(f^{*}\right)$, there exists $h \in C(X)$ such that $Z\left(h^{*}\right) \supseteq K$ and $p \notin \operatorname{cl}_{\beta X} Z(h)$. Since $\beta X \sim Z\left(f^{*}\right)$ is an $F_{\sigma}$ in $\beta X$, it is Lindelöf, and therefore we can find $g_{1}, g_{2}, g_{3}, \cdots \in C(X)$ such that $Z\left(g_{n}^{*}\right) \supseteq K$ for each $n$, and $\bigcap_{n=1}^{\infty} \operatorname{cl}_{\beta X} Z\left(g_{n}\right) \subseteq Z\left(f^{*}\right)$. Defining $g=\sum_{n=1}^{\infty}\left(1 / 2^{n}\right)\left(\left|g_{n}\right| \wedge 1\right) \in C(X)$, we have $Z(g)=\bigcap_{n=1}^{\infty} Z\left(g_{n}\right) \subseteq Z(f)$ and $Z\left(g^{*}\right)=\bigcap_{n=1}^{\infty} Z\left(g_{n}^{*}\right) \supseteq K$. It follows that $\mathrm{cl}_{\beta X} Z(f) \supseteq \mathrm{cl}_{\beta X} Z(g) \supseteq \Delta$.

4. Some corollaries. We now consider some consequences of 3.1 and 3.2. The first result follows also from $[\mathbf{P}, 2.6]$, where a more algebraic proof is given.

4.1 Corollary. If the ideal I in $C(X)$ is contained in a unique maximal ideal (e.g., if I is prime), then I is closed if and only if $I$ is a real ideal.

Proof. Clearly a real ideal is closed [GJ, 8.4]. Suppose that $I$ is a closed ideal which is contained in the unique maximal ideal $M^{p}$ for some $p \in \beta X$. Then $O^{p} \subseteq I$ [GJ, 7.13], from which it follows that $\bigcap Z\left[I^{*}\right]=\{p\}$. Thus, $\{p\}$ is an ideal set, and it follows from 3.1 that, if $q \in \beta X \sim v X$, then $\operatorname{cl}_{\beta X}(\{p\} \sim\{q\})=\{p\}$-i.e., $q \neq p$. Hence $p \in v X$ and $I=M^{p}$, a real ideal.

It is clear that, for any nonvoid $E \subseteq X$, the set $\mathrm{cl}_{\beta X} E$ is an ideal set. 
The next result characterizes those $X$ for which all ideal sets are of this form. It can also be deduced from [P, 3.3 and 3.4].

4.2 COROLlaRY. The following conditions are mutually equivalent.

(a) $X$ is Lindelöf.

(b) Every ideal set in $\beta X$ is of the form $\mathrm{cl}_{\beta X} E$ for some $E \subseteq X$.

(c) Every ideal set in $\beta X$ meets $X$.

(d) Every closed ideal in $C(X)$ is an intersection of fixed maximal ideals.

(e) Every closed ideal in $C(X)$ is fixed.

Proof. . (a) implies (b). Assume (a), let $\Delta$ be an ideal set in $\beta X$, and suppose that $p \in \beta X \sim \mathrm{cl}_{\beta X}(\Delta \cap X)$. Let $F$ be a closed neighborhood of $p$ such that $F \cap(\Delta \cap X)=\varnothing$. Then $S=F \cap \Delta$ is a compact subset of $\beta X \sim X$, and hence by $3.1(\mathrm{~d}), p \notin \mathrm{cl}_{\beta X}(\Delta \sim S)=\Delta$. Hence $\Delta=\mathrm{cl}_{\beta X}(\Delta \cap X)$, and (b) holds.

(b) implies (c). Obvious.

(c) implies (a). Assume that (a) is false, so there exists a compact subset $K$ of $\beta X \sim X$ which is close to $X$. By 3.2, $K$ contains an ideal set $\Delta$, so that $(c)$ is false.

(b) if and only if (d). This follows easily from the fact that, for any $E \subseteq X, f \in \bigcap\left\{M^{p}: p \in E\right\}$ if and only if $\operatorname{cl}_{\beta X} E \subseteq Z\left(f^{*}\right)$.

(c) if and only if (e). A closed ideal $\left\{f \in C(X): Z\left(f^{*}\right) \supseteq \Delta\right\}$ is fixed if and only if $\Delta$ meets $X$.

In 4.1 we showed that an ideal in $C(X)$ is a closed maximal ideal if and only if it is real. Clearly every closed maximal ideal is a maximal closed ideal. We conclude by proving the converse.

4.3 Corollary. An ideal in $C(X)$ is a closed maximal ideal if and only if it is a maximal closed ideal.

Proof. It suffices to show that every maximal closed ideal is a maximal ideal. Thus, suppose that $I$ is a maximal closed ideal in $C(X)$, and let $\Delta=\bigcap Z\left[I^{*}\right]$. If $\Delta$ is not a singleton set, say $q_{1}, q_{2} \in \Delta$ with $q_{1} \neq q_{2}$, then there exist compact sets $K_{1}$ and $K_{2}$ such that $K_{1} \cup K_{2}=\Delta, q_{1} \notin K_{1}$ and $q_{2} \notin K_{2}$. At least one of $K_{1}$ and $K_{2}$, say $K_{1}$, must be close to $X$. By $3.2, K_{1}$ contains an ideal set $\Delta_{1}$. But since $q_{1} \in \Delta \sim \Delta_{1}$, the closed ideal $J=$ $\left\{f \in C(X): Z\left(f^{*}\right) \supseteq \Delta_{1}\right\}$ is strictly bigger than $I$, contradicting the maximality of $I$. Therefore, we must have $\Delta=\{p\}$ for some $p \in \beta X$. But then $p \in v X$, and $I=M^{p}$, a maximal ideal.

\section{REFERENCES}

[GJ] L. Gillman and M. Jerison, Rings of continuous functions, University Series in Higher Math., Van Nostrand, Princeton, N.J., 1960. MR 22 \#6994.

[H] E. Hewitt, Rings of real-valued continuous functions. I, Trans. Amer. Math. Soc. 64 (1948), 45-99. MR 10, 126. 
[P] D. Plank, Closed l-ideals in a class of lattice-ordered algebras, Illinois J. Math. 15 (1971), 515-524.

[S] Ju. M. Smirnov, On normally disposed sets of normal spaces, Mat. Sb. 29 (71) (1951), 173-176. (Russian) MR 13, 371.

Department of Mathematics, St. Mary's College of Maryland, St. Mary's City, Maryland 20686 (Current address of Philip Nanzetta)

Department of Mathematics, Amherst College, AmHerst, Massachusetts 01002

Current address (Donald Plank): Department of Mathematics, Stockton State College, Pomona, New Jersey 08240 